\title{
Patterns of Lumbar Disc Degeneration: Magnetic Resonance Imaging Analysis in Symptomatic Subjects
}

\author{
Monchai Ruangchainikom ${ }^{1,2}$, Michael D. Daubs ${ }^{3}$, Akinobu Suzuki ${ }^{4}$, \\ Chengjie Xiong ${ }^{5}$, Tetsuo Hayashi ${ }^{6}$, Trevor P. Scott ${ }^{7}$, Kevin Phan $^{8}$, Jeffrey C. Wang ${ }^{9}$ \\ ${ }^{1}$ Department of Orthopedic Surgery, Faculty of Medicine Siriraj Hospital, Mahidol University, Bangkok, Thailand \\ ${ }^{2}$ Department of Orthopedic Surgery, University of California Los Angeles, Los Angeles, CA, USA \\ ${ }^{3}$ Department of Orthopedic Surgery, School of Medicine, University of Nevada Las Vegas, Las Vegas, NV, USA \\ ${ }^{4}$ Department of Orthopaedic Surgery, Faculty of Medicine, Osaka City University, Osaka, Japan \\ ${ }^{5}$ Department of Orthopaedic Surgery, General Hospital of the Central Theater Command of the People's Liberation Army, Wuhan, China \\ ${ }^{6}$ Department of Orthopaedic Surgery, Japan Organization of Occupational Health and Safety, Spinal Injuries Center, Fukuoka, Japan \\ Proliance Orthopedics and Sports Medicine, Bellevue, WA, USA \\ ${ }^{8}$ Department of Orthopedic Surgery, Montefiore Medical Center, New York, NY, USA \\ ${ }^{9}$ USC Spine Center, Department of Orthopedic Surgery, Faculty of Medicine, University of Southern California, Los Angeles, CA, USA
}

Study Design: Cross-sectional study.

Purpose: To evaluate lumbar disc degeneration (LDD) on magnetic resonance imaging (MRI) in symptomatic subjects to accumulate baseline data on the pattern of degeneration.

Overview of Literature: LDD plays an important role in the diagnosis and treatment of low-back pain in patients. Few studies have focused on the pattern of LDD to understand how the lumbar spine ages.

Methods: This study included 1,095 patients (mean age, 44.29 years; range, 16-85 years) who underwent upright lumbar MRI. LDD was graded into five categories (I-V). Positive LDD was defined as grade III or greater. The prevalence and pattern of LDD were analyzed, and the correlations between age and total grade of LDD were evaluated.

Results: The average number of LDD levels and the total grade of LDD increased with age. LDD moved cephalad with age. The rate of LDD increased rapidly during the decade before the prevalence of LDD and became $>50 \%$. In the single-level LDD group, the levels L5-S1 were the most common levels (60.3\%). In the two-level group, L4-L5 and L5-S1 were the most common levels (53.5\%). In the three-level group, L3-L4, L4-L5, and L5-S1 were the most common levels (55.7\%). In the multilevel LDD group, contiguous multilevel disc degeneration (CMDD) was more common than the skipped level disc degeneration (SLDD). The levels L4-L5 were the most common levels in the CMDD group, and L5-S1 were the most common levels among SLDD.

Conclusions: LDD was found to correlate with age, and the specific patterns and rates of LDD depended on lumbar disc level and age. These LDD pattern data can be used before spinal procedures to predict the probability of natural LDD progression with age.

Keywords: Intervertebral disc degeneration; Magnetic resonance imaging; Low back pain; Patterns of degeneration

Received Jun 25, 2020; Revised Sep 7, 2020; Accepted Oct 7, 2020

Corresponding author: Monchai Ruangchainikom

Department of Orthopedic Surgery, Faculty of Medicine Siriraj Hospital, Mahidol University, 2 Wanglang Road, Bangkoknoi, Bangkok 10700, Thailand

Tel: +66-2-419-7958, Fax: +66-2-419-7967, E-mail: monchai.rua@mahidol.ac.th 


\section{Introduction}

Intervertebral lumbar disc degeneration (LDD) is a common finding on magnetic resonance imaging (MRI). The prevalence of LDD was reported to range from $26 \%-91 \%$ depending on the age of the patient population [1-5].

The reported association between low-back pain and LDD ranged from mild to strong $[1,3,6,7]$. Lumbar spinal fusion is the mainstay spine procedure for treatment of patients with lumbar diseases. However, adjacent segment disease (ASD) is a condition that often develops after fusion procedure. The prevalence of ASD ranged from lower than $10 \%$ to nearly $100 \%$ [8-10]. Many different motionsparing procedures have been developed to reduce the incidence of ASD [11,12]. A recent meta-analysis conducted by Pan et al. [13] investigated the efficacy of these procedures to reduce the prevalence of ASD compared with lumbar fusion. However, the rate of ASD was still as high as $18.6 \%$. The cause of ASD was reported to be a combination of processes related to both biomechanical stress and the natural progression of the disease $[9,14,15]$. The relative scarcity of data, and the observed variations in the reported evidence make it difficult to develop a conclusive understanding of LDD.

In addition to the understanding the structural changes of LDD, we must also learn and understand the pattern of LDD relative to the number of levels of LDD, and the combinations of lumbar levels involved. This information will help us predict the pattern of LDD, and may improve our ability to customize treatment to the needs of individual patients.

MRI is the gold standard investigation for evaluating LDD. MRI demonstrated an ability to precisely evaluate LDD, and to yield information needed to accurately and reliably classify the severity of LDD [16]. The aim of this study was to evaluate LDD on MRI in a large cohort of symptomatic subjects with low-back pain to accumulate baseline data on the pattern of LDD to better understand how the lumbar spine ages.

\section{Materials and Methods}

\section{Patient population}

This cross-sectional study included patients who were referred for upright MRI owing to complaints of lowback pain with or without leg pain. The Institutional
Review Board of the University of California Los Angeles approved this study (approval no., 10-000968), and informed consent was obtained from all participants.

\section{Magnetic resonance imaging}

A total of 1,169 patients (507 females, 662 males) were included with a mean age of 44.29 years (range, 16-85 years). Patients with a previous history of spinal surgery or vertebral fracture, or who had incomplete or poor imaging outcomes were excluded.

MRI of the lumbar spine was performed using a 0.6 Tesla MRI scanner (UPRIGHT Multi-Position; Fonar Corp., Melville, NY, USA). Two vertically oriented opposing magnetic doughnuts placed $45.72 \mathrm{~cm}$ apart were used that facilitated scanning of the patients in an upright, axially loaded position. A planar quadrature channel radiofrequency coil was used to acquire images. We examined the longitudinal relaxation (T1)-weighted sagittal spin echo images (repetition time, $671 \mathrm{~ms}$; echo time, $17 \mathrm{~ms}$; slice thickness, $4.0 \mathrm{~mm}$; field-of-view, $30 \mathrm{~cm}$; matrix, 256×224; number of excitations [NEX], 2) and transverse relaxation (T2)-weighted fast spin echo images (repetition time, 3,000 $\mathrm{ms}$; echo time, $140 \mathrm{~ms}$; thickness, $4.0 \mathrm{~mm}$; field-of-view, $30 \mathrm{~cm}$; matrix, 256×224; NEX, 2) for all patients.

\section{Grading system for lumbar disc degeneration}

We measured all functional lumbar spinal levels, including L1-L2, L2-L3, L3-L4, L4-L5, and L5-S1. Four spine surgeons graded the degree of disc degeneration into five grades using T2-weighted neutral sagittal images according to the grading system proposed by Pfirrmann et al. [16] (Table 1).

Intraobserver and interobserver agreement for grading of the included intervertebral discs was determined using MRI from 100 randomly selected subjects. The reliability of MRI grading was estimated using agreement percentages and kappa statistics for each observer (intraobserver reliability), and among the four spine surgeons (interobserver reliability).

\section{Analysis of lumbar disc degeneration grade}

A total of 5,845 lumbar intervertebral discs were classified into five grades according to the criteria set forth in the Pfirrmann grading system by the same four spine 


\begin{tabular}{lllll} 
Table 1. Disc degeneration grading system & Structure & Sistinction of \\
Grade & $\begin{array}{c}\text { nucleus and } \\
\text { annulus }\end{array}$ & Signal intensity & Height of intervertebral disc \\
I & Homogeneous, bright white & Clear & Hyperintense, isointense to cerebrospinal fluid & Normal \\
II & Inhomogeneous with or without horizontal bands & Clear & Hyperintense, isointense to cerebrospinal fluid & Normal \\
III & Inhomogeneous, grey & Unclear & Intermediate & Normal to slightly decreased \\
IV & Inhomogeneous, grey to black & Lost & Intermediate to hypointense & Normal to moderately decreased \\
V & Inhomogeneous, black & Lost & Hypointense & Collapsed disc \\
\hline
\end{tabular}

surgeons [16]. The prevalence of degenerative disc grading at each lumbar level was assessed. The total LDD (total LDD) score was calculated by summarizing the degenerative disc score of all lumbar levels (L1-L2, L2-L3, L3L4, L4-L5, and L5-S1) in each patient. The correlation between total LDD and age was evaluated. Age decade was defined as follows: 10s, 11-20 years; 20s, 21-30 years; 30 s, $31-40$ years; 40 s, $41-50$ years; 50 s, $51-60$ years; 60 s, $61-70$ years; and $70 s, \geq 71$ years.

Discs that were classified as Pfirrmann grades I or II were defined as nonlumbar disc degeneration states. Alternatively, discs that were graded as Pfirmann grade III, IV, or V were defined as significant LDD states. The number of lumbar levels of LDD was also assessed. Accordingly, patients were classified as single-, two-, three-, four-, or five-level LDDs. The prevalence of the number of levels of LDD was also analyzed.

Multilevel lumbar disc degeneration (more than two levels of disc degeneration) was further classified as skipped level lumbar disc degeneration (SLDD) or contiguous multilevel lumbar disc degeneration (CMDD). SLDD was defined as skipped or noncontiguous, multilevel involvement, and CMDD was defined as multilevel involvement that affects immediately adjacent lumbar levels [3]. The prevalence of SLDD and CMDD was assessed based on the number of levels affected, and the specific combinations of levels affected.

\section{Statistical analysis}

All statistical analyses were performed using IBM SPSS software ver. 20.0 (IBM Corp., Armonk, NY, USA). Pearson's correlation coefficient was used to analyze the correlation between the patient age and total disc degeneration score, number of levels of disc degeneration, and degree of disc degeneration. The pattern of disc degeneration was

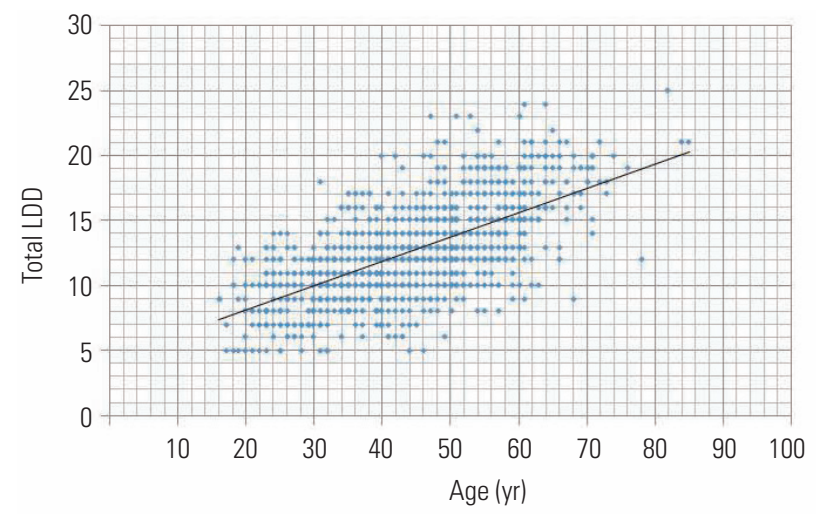

Fig. 1. Plot showing moderate correlation between total LDD score and age (Pearson's correlation coefficient: 0.649). LDD, lumbar disc degeneration.

analyzed using chi-square tests. Categorical data are presented as number and percentage. The level of statistical significance was set at $p<0.05$.

\section{Results}

\section{Interobserver and intraobserver agreement}

Intraobserver agreement of the four observers was excellent with kappa values ranging from 0.84 to 0.94 . Interobserver agreement among the four reviewers was good to excellent with kappa values ranging from 0.77 to 0.90 .

\section{Degree of lumbar disc degeneration and age}

Total disc degeneration score showed a moderate positive correlation with increasing age (Pearson's correlation coefficient: $0.649 ; p<0.001$ ) (Fig. 1). The degree of degeneration at each lumbar level exhibited mild to moderate correlation with aging (Pearson's correlation coefficients: L1-L2, 0.42; L2-L3, 0.49; L3-L4, 0.55; L4-L5, 0.48; and L5-S1, 0.36; $p<0.001)$. Moderate correlation was observed 


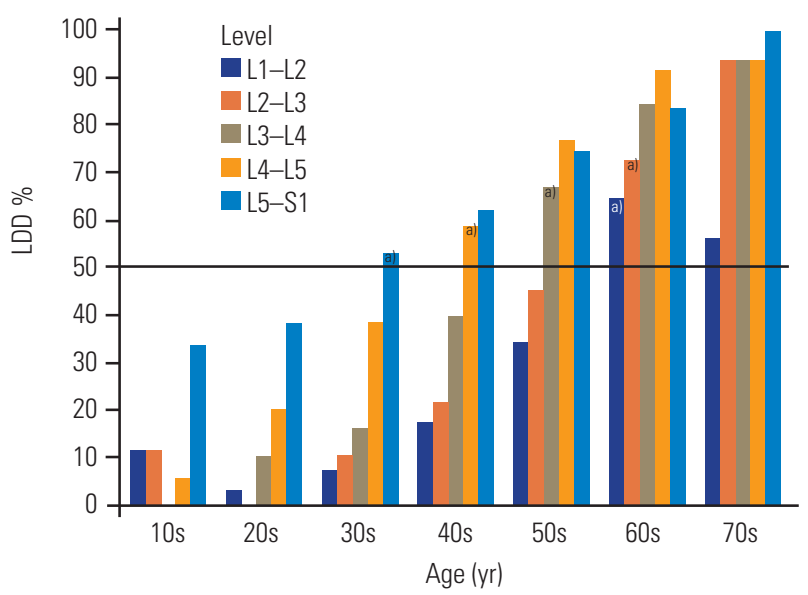

Fig. 2. Percentage of LDD compared among lumbar levels by the age by de-

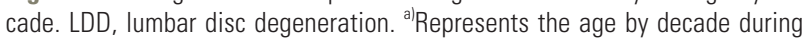
which the percentage of LDD for that lumbar disc level became greater than $50 \%$.

between aging and the number of affected lumbar levels (Pearson's correlation coefficient: $0.60 ; p<0.5$ ).

\section{Rates of lumbar disc degeneration}

Regarding the percentage of LDD at each lumbar level, the percentages tended to increase with age for all lumbar levels (Fig. 2). Caudad levels degenerated earlier than cephalad levels. Specifically, the data showed that the percentage of LDD was $>50 \%$ at an in earlier age at the caudad levels than at the cephalad level (i.e., L5-S1 during the 30s, L4L5 during the 40s, L3-L4 during the 50s, and L1-L2 and L2-L3 during the 60s).

Interestingly, the rates of disc degeneration increased rapidly during the decade before the prevalence of LDD became greater than $50 \%$ for all lumbar levels. L1-L2 and L2-L3 from the 50 s to the 60 s had the highest percentage increase in disc degeneration $30.47 \%$ and $27.56 \%$, sequentially). The percentage of LDD at L3-L4 increased rapidly $(26.78 \%)$ from the 40 s to the 50 s. The LDD at L4L5 increased rapidly (20.36\%) from the 30 s to the 40 s. LDD at L5-S1 increased markedly (14.85\%) from the 20 s to the 30s. The greatest increase in LDD at L5-S1 was observed from the 60 s to the 70 s (16.47\%).

\section{Number of lumbar disc degeneration levels and age}

LDD started as early as the first decade of life, and these individuals had LDD less than $50 \%$ after their 20 s. The prevalence of single-level LDD increased from the first

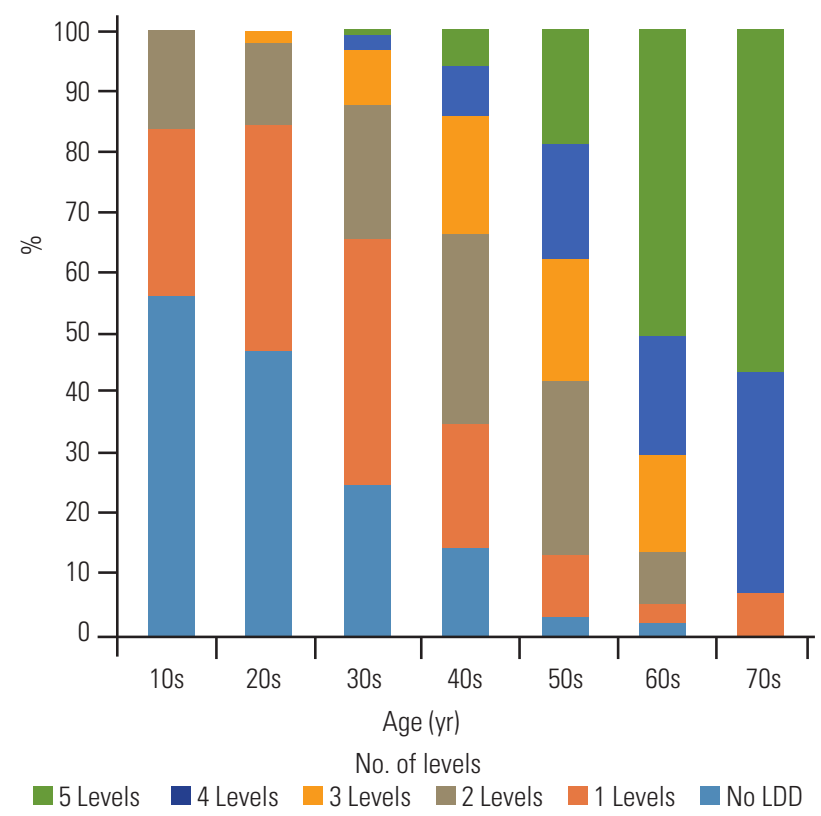

Fig. 3. Distribution of the number of levels of LDD according to the age by decade. LDD, lumbar disc degeneration.

decade of life to its peak during the 30s. Two-level LDD increased gradually from young age to its peak during the $50 \mathrm{~s}$, and then there was a sudden decrease during the 60s. Three-level LDD started from the 20s to its peak during the 50s, and then three-level LDD became greater than two-level LDD during their 60s and 70s. Four-level LDD started during the third decade of life and increased slowly to the sixth decade, and then rapidly increased during the seventh decade. Five-level LDD started during the 30 s and increased rapidly from the 50 s to the 60 s. Greater than $50 \%$ of our study population had been diagnosed with five-level LDD at ages $>60$ years (Fig. 3).

In single-level LDD, the most common level was L5-S1. In multilevel LDD, the highest percentage increase moved cephalad from L4-L5 to L1-L2, in addition to a singlelevel increase in disc degeneration (Table 2). In two-level LDD, the highest increasing percentage from one-level LDD (49.01\%) occurred at L4-L5. In three-level LDD, the highest percentage increase from two-level LDD (47.09\%) was observed at L3-L4. In four-level LDD, the highest increasing percentage from three-level LDD (49.57\%) took place at L2-L3. In five-level LDD, the highest percentage increase from four-level LDD (50.49\%) was identified at L1-L2. Among multilevel LDD, CMDD $(\mathrm{n}=485)$ was more common than SLDD ( $\mathrm{n}=131)$ (ratio, 7.3:2.7) (Table $3)$. 
Table 2. Distribution of the number of levels of LDD compared among different levels of lumbar intervertebral disc involvement

\begin{tabular}{lccccc} 
No. of levels & L1-L2 & L2-L3 & L3-L4 & L4-L5 & L5-S1 \\
\hline Single-level LDD (n=282) & $10(3.55)$ & $4(1.42)$ & $25(8.87)$ & $73(25.89)$ & $170(60.28)$ \\
\hline Two-level LDD (n=243) & $24(9.88)$ & $26(10.7)$ & $78(32.1)$ & $182(74.9)$ & $176(72.43)$ \\
\hline Three-level LDD (n=149) & $24(16.11)$ & $52(34.9)$ & $118(79.19)$ & $129(86.58)$ & $124(83.22)$ \\
\hline Four-level LDD (n=103) & $51(49.51)$ & $87(84.47)$ & $95(92.23)$ & $97(94.17)$ & $82(79.61)$ \\
\hline Five-level LDD (n=121) & $121(100)$ & $121(100)$ & $121(100)$ & $121(100)$ & $121(100)$ \\
\hline
\end{tabular}

Values are presented as number (\%). The greatest percentage increase when each level had a one-level increase in degeneration is in boldface. LDD, lumbar disc degeneration.

Table 3. Multilevel-LDD patterns compared between CMDD and SLDD

\begin{tabular}{|c|c|c|c|c|}
\hline Variable & CMDD & No. $(\%)$ & SLDD & №. $(\%)$ \\
\hline \multirow[t]{7}{*}{ Two-level LDD (n=243) } & L4-L5, L5-S1 & $130(53.5)$ & L3-L4, L5-S1 & 28 (11.52) \\
\hline & L3-L4, L4-L5 & $42(17.28)$ & $\mathrm{L} 1-\mathrm{L} 2, \mathrm{~L} 5-\mathrm{S} 1$ & $11(4.53)$ \\
\hline & $\mathrm{L} 1-\mathrm{L} 2, \mathrm{~L} 2-\mathrm{L} 3$ & $7(2.88)$ & L2-L3, L5-S1 & $7(2.88)$ \\
\hline & L2-L3, L3-L4 & $6(2.47)$ & $\mathrm{L} 2-\mathrm{L} 3, \mathrm{~L} 4-\mathrm{L} 5$ & $6(2.47)$ \\
\hline & - & - & $\mathrm{L} 1-\mathrm{L} 2, \mathrm{~L} 4-\mathrm{L} 5$ & $4(1.65)$ \\
\hline & - & - & $\mathrm{L} 1-\mathrm{L} 2, \mathrm{~L} 3-\mathrm{L} 4$ & $2(0.82)$ \\
\hline & Two-level CMDD & $185(76.13)$ & Two-level SLDD & $58(23.87)$ \\
\hline \multirow[t]{8}{*}{ Three-level LDD ( $n=149)$} & L3-L4, L4-L5, L5-S1 & $83(55.7)$ & L2-L3, L4-L5, L5-S1 & 16 (10.74) \\
\hline & L2-L3, L3-L4, L4-L5 & $18(12.08)$ & L1-L2, L4-L5, L5-S1 & $10(6.71)$ \\
\hline & L1-L2, L2-L3, L3-L4 & $5(3.36)$ & L2-L3, L3-L4, L5-S1 & $8(5.37)$ \\
\hline & - & - & L1-L2, L3-L4, L5-S1 & $4(2.68)$ \\
\hline & - & - & $\mathrm{L} 1-\mathrm{L} 2, \mathrm{~L} 2-\mathrm{L} 3, \mathrm{~L} 5-\mathrm{S} 1$ & $3(2.01)$ \\
\hline & - & - & L1-L2, L2-L3, L4-L5 & $2(1.34)$ \\
\hline & - & - & L1-L2, L3-L4, L4-L5 & 0 \\
\hline & Three-level CMDD & $106(71.14)$ & Three-level SLDD & 43 (28.86) \\
\hline \multirow[t]{4}{*}{ Four-level LDD (n=103) } & L2-L3, L3-L4, L4-L5, L5-S1 & $52(50.48)$ & $\mathrm{L} 1-\mathrm{L} 2, \mathrm{~L} 3-\mathrm{L} 4, \mathrm{~L} 4-\mathrm{L} 5, \mathrm{~L} 5-\mathrm{S} 1$ & 16 (15.53) \\
\hline & L1-L2, L2-L3, L3-L4, L4-L5 & $21(20.39)$ & $\mathrm{L} 1-\mathrm{L} 2, \mathrm{~L} 2-\mathrm{L} 3, \mathrm{~L} 4-\mathrm{L} 5, \mathrm{~L} 5-\mathrm{S} 1$ & $8(7.77)$ \\
\hline & - & - & $\mathrm{L} 1-\mathrm{L} 2, \mathrm{~L} 2-\mathrm{L} 3, \mathrm{~L} 3-\mathrm{L} 4, \mathrm{~L} 5-\mathrm{S} 1$ & $6(5.83)$ \\
\hline & Four-level CMDD & 73 (70.87) & Four-level SLDD & $30(29.13)$ \\
\hline
\end{tabular}

LLD, lumbar disc degeneration; CMDD, contiguous-multilevel disc degeneration; SLDD, skip level disc degeneration.

Among the CMDD group, the most common combinations were among the caudad levels, as follows: L4-L5 and L5-S1 (53.50\%) in two-level LDD; L3-L4, L4-L5, and L5S1 (55.70\%) in three-level LDD; and, L2-L3, L3-L4, L4L5, and L5vS1 (50.48\%) in four-level LDD. Interestingly, the most common lumbar disc level in CMDD was L4-L5 (96.28\%), not L5-S1 (54.64\%).

In SLDD, the most common combinations were the combination of caudad levels with one normal level preserved disc next to the uppermost degenerative disc, as follows: L3-L4 and L5-S1 (11.52\%) in two-level LDD; L2-L3, L4-L5, and L5-S1 (10.74\%) in three-level LDD; and L1-L2, L3-L4, L4-L5, and L5-S1 (15.53\%) in fourlevel LDD. The most common LDD in SLDD was L5-S1 (89.31\%).

\section{Discussion}

This cross-sectional study showed baseline of patterns of LDD in symptomatic patients, and the characteristics of 
LDD - mainly specific to lumbar level-were reported. These findings may help surgeons to understand the patterns of LDD in symptomatic patients. Correspondingly, this information may enhance individual treatment planning and improve patient outcomes.

Aging is a factor that is commonly reported to be associated with disc degeneration [2,3,17-20]. Consistent with these findings, the present study also found that aging was significantly associated with the total LDD score. In addition to total LDD score, we also found aging to be associated with both degree of disc degeneration and number of levels of LDD. The decade of age when the percentage of LDD became greater than 50\% (L5-S1 during the 30s, L4-L5 during the 40s, L3-L4 during the 50s, and L1-L2 and L2-L3 during the 60s) should be noted because of the high acceleration of LDD. To the best of our knowledge, the present study is the first to report the high acceleration of disc degeneration during the decade of life before the prevalence of LDD at that level became greater than 50\% in symptomatic patients. The aforementioned results support the fact that aging plays an important role in LDD. This LDD pattern finding may help clinicians to assess the level of patient risk according to the patient's age group, and may further enhance treatment-related decisionmaking. We also compared our symptomatic study to the 2013 asymptomatic study by Kim et al. [4] that reported the prevalence of asymptomatic LDD in patients with a mean age 46.3 years. These authors based their grading system on signal intensities. Therefore, their prevalence results tended to be close to our prevalence rates during the decade in which $50 \%$ of patients had LDD. That group also reported the acceleration of disc degeneration in the decade that preceded the decade in which the prevalence of LDD reached $50 \%$. Compared with our results, Kim et al. [4] reported a less consistent prevalence slope for L5S1 LDD; nevertheless, their L5-S1 LDD prevalence was higher than the other levels in the younger age groups. The same pattern of the progression of LDD in asymptomatic cases in the study by Kim et al. [4] and symptomatic patients in our study may support the natural program of LDD processes.

Data from the present study also showed involvement of different lumbar levels in the cases associated with the highest prevalence of LDD when single- and multiple-level degenerations were compared. The lumbar and sacral levels with the high prevalence of LDD in single-level LDD were L5-S1. However, the L4-L5 levels were slightly more common than the L5-S1 levels in multilevel LDD. This observed difference between single-level and multiple-level LDD can be explained. L5-S1 is the lumbosacral junction comprising the last levels to receive compression loads. Thus, the chance of this level being the first level to develop LDD is high. This may also be the reason why the prevalence of LDD in single-level LDD is the highest at the levels of L5-S1 among younger aged patients. When patients become older, multilevel LDD is more common. CMDD was predominant at L4-L5, whereas SLDD was predominant at L5-S1. Levels L5-S1 will less likely to combine with lumbar levels (other than L4-L5) in CMDD types. However, L4-L5 has two adjacent levels to combine with. This is why the chance of their combination with another level is higher compared with L5S1. Another reason may be anatomic protective factors, such as lumbosacral transition, iliolumbar ligament, and high-intercrestal line, because these factors were found to protect some patients from mechanical cause of disc degeneration at L5-S1, whereas L4-L5 is not associated with any similar protective factors [21-24]. Studies often report their results without describing in detail the number of levels of LDD and the ratio of CMDD to SLDD. This may be one of the reasons that a variety of lumbar levels (commonly L4-L5 or L5-S1) for which they exhibit the highest LDD prevalence in different age groups $[5,19,25,26]$. If the study population predominantly consists of younger patients, LDD will be dominant at L5-S1, and most cases will be single-level LDD. However, if most of these cases are at an advanced age, L4-L5 will be the dominant level of LDD, and there will be a high prevalence of CMDD.

The common pattern of multiple disc degeneration was contiguous level $(76.13 \%$ in the two-level degeneration group, $71.14 \%$ in the three-level group, and $70.87 \%$ in the four-level group). The degeneration usually occurs at the adjacent level, as shown in previous biomechanics and clinical studies. Natarajan and Andersson [27] conducted a biomechanical study using finite element analysis that showed the effect of degenerative disc on the sagittal angular motion at the adjacent level. Our previous study also demonstrated an adverse effect of the degenerative disc on the sagittal angular motion as evaluated by kinematic MRI [28]. The study by Cheung et al. [29] on intervertebral disc degeneration found CMDD to be far more prevalent than SLDD among studied volunteers of Southern Chinese origin with a CMDD to SLDD ratio of 7.9:2.1. In our study, the ratio of CMDD to SLDD was 7.3:2.7 for multi- 
level LDD in symptomatic patients. These findings seem to suggest that the CMDD to SLDD ratio may be the same in different populations. Nevertheless, these two different study populations cannot be directly compared, so this hypothesis requires further study.

The commonly observed pattern among SLDD involved the skipping of one level of nondegenerative intervertebral disc with a combination of lowermost caudad lumbar level LDD, as follows: two-level SLDD: L3-L4 and L5-S1, three-level SLDD: L2-L3, L4-L5, and L5-S1, and four-level SLDD: L1-L2, L3-L4, L4-L5, and L5-S1. The risk factors for SLDD that were reported were male, had a history of back injury, had a documented presence of Schmorl's node and a bulging disc [29]. Increasing prevalence of upper and midlumbar (L1-L2, L2-L3, and L3L4) level LDD was detected in this subtype. Furthermore, preexisting injury and/or coexisting defect or pathology were more often observed in patients with LDD at higher lumbar levels [30]. Genetic and familial influences were factors reported to be significantly associated with disc degeneration, and lifetime occupations that require heavy lifting and leisure physical loading were identified as adjunct factors that increase the chance of upper lumbar LDD [25]. SLDD should be determined and the risk factors should be evaluated in patients who plan to undergo spine surgery.

The LDD findings from the present study should be used to further study the degeneration of adjacent segments to differentiate natural progression from mechanical aggravation. An adjacent segment degeneration study would also benefit from the inclusion of additional data, including the number of levels, CMDD or SLDD, and common or uncommon subtypes. Moreover, the proposed analysis must include both adjacent and all other lumbar levels to determine patterns of activity. Lastly, in contrast to our study that included mostly middle-aged patients, a future study on adjacent segment degeneration should include data that reflects a balance among different age groups.

This study was associated with limitations. First, this was a cross-sectional study-not a longitudinal study, which would have higher accuracy for determining patterns of LDD. Second, the population enrolled in this study was predominantly middle age, so our results may not reflect or be generalizable to other age groups of symptomatic patients. Third, there were many changing structures that influenced the pattern of disc degenera- tion, such as endplate changes, facet joint degeneration, facet tropism, Schmorl's nodes, and high-intensity zone, and these factors were not analyzed in this study. Lastly, we did not evaluate or analyze the association between MRI finding and clinical data specific to the degree of patient symptoms attributed to the lack of clinical data specific to low-back pain based on a standard scoring system. The strength of this study is that it provides baseline information from a large cohort of symptomatic patients that can be used for comparison with future cases to improve treatment planning.

\section{Conclusions}

This cross-sectional study used MRI to determine the prevalence of natural patterns of LDD in symptomatic middle-aged patients. LDD was more common in the lower lumbar spine, and the number of levels of LDD and the degree of LDD increased with age. The specific pattern and rate of LDD depended on the lumbar disc level and age. This LDD pattern data can be used before spinal procedures to predict the likelihood of natural LDD progression with age, and this preoperative assessment will enhance treatment planning and outcomes.

\section{Conflict of Interest}

No potential conflict of interest relevant to this article was reported.

\section{Acknowledgments}

The authors gratefully acknowledge Miss Nhathita Panatreswas for statistical analysis.

\section{ORCID}

Monchai Ruangchainikom: https://orcid.org/0000-00030525-6390; Michael D. Daubs: https://orcid.org/0000-00020912-315X; Akinobu Suzuki: https://orcid.org/0000-00034800-9226; Chengjie Xiong: https://orcid.org/0000-00030044-4499; Tetsuo Hayashi: https://orcid.org/0000-00021015-4922; Trevor P. Scott: https://orcid.org/0000-00022484-2160; Kevin Phan: https://orcid.org/0000-0001-79367830; Jeffrey C. Wang: https://orcid.org/0000-0002-62977410 


\section{References}

1. Tertti MO, Salminen JJ, Paajanen HE, Terho PH, Kormano MJ. Low-back pain and disk degeneration in children: a case-control MR imaging study. Radiology 1991;180:503-7.

2. Jarvik JJ, Hollingworth W, Heagerty P, Haynor DR, Deyo RA. The Longitudinal Assessment of Imaging and Disability of the Back (LAIDBack) Study: baseline data. Spine (Phila Pa 1976) 2001;26:1158-66.

3. Cheung KM, Karppinen J, Chan D, et al. Prevalence and pattern of lumbar magnetic resonance imaging changes in a population study of one thousand fortythree individuals. Spine (Phila Pa 1976) 2009;34:93440.

4. Kim SJ, Lee TH, Lim SM. Prevalence of disc degeneration in asymptomatic Korean subjects. Part 1: lumbar spine. J Korean Neurosurg Soc 2013;53:31-8.

5. Takatalo J, Karppinen J, Niinimaki J, et al. Prevalence of degenerative imaging findings in lumbar magnetic resonance imaging among young adults. Spine (Phila Pa 1976) 2009;34:1716-21.

6. MacGregor AJ, Andrew T, Sambrook PN, Spector TD. Structural, psychological, and genetic influences on low back and neck pain: a study of adult female twins. Arthritis Rheum 2004;51:160-7.

7. Chou D, Samartzis D, Bellabarba C, et al. Degenerative magnetic resonance imaging changes in patients with chronic low back pain: a systematic review. Spine (Phila Pa 1976) 2011;36(21 Suppl):S43-53.

8. Bae JS, Lee SH, Kim JS, Jung B, Choi G. Adjacent segment degeneration after lumbar interbody fusion with percutaneous pedicle screw fixation for adult low-grade isthmic spondylolisthesis: minimum 3 years of follow-up. Neurosurgery 2010;67:1600-8.

9. Ishihara H, Osada R, Kanamori M, et al. Minimum 10-year follow-up study of anterior lumbar interbody fusion for isthmic spondylolisthesis. J Spinal Disord 2001;14:91-9.

10. Xia XP, Chen HL, Cheng HB. Prevalence of adjacent segment degeneration after spine surgery: a systematic review and meta-analysis. Spine (Phila Pa 1976) 2013;38:597-608.

11. Han Y, Sun J, Luo C, et al. Comparison of pedicle screw-based dynamic stabilization and fusion surgery in the treatment of radiographic adjacent-segment degeneration: a retrospective analysis of single L5-S1 degenerative spondylosis covering 4 years. J Neurosurg Spine 2016;25:706-12.

12. Ciplak NM, Suzer T, Senturk S, et al. Complications of 2-level dynamic stabilization: a correlative clinical and radiological analysis at two-year follow-up on 103 patients. Turk Neurosurg 2018;28:756-62.

13. Pan A, Hai Y, Yang J, Zhou L, Chen X, Guo H. Adjacent segment degeneration after lumbar spinal fusion compared with motion-preservation procedures: a meta-analysis. Eur Spine J 2016;25:1522-32.

14. Eck JC, Humphreys SC, Hodges SD. Adjacent-segment degeneration after lumbar fusion: a review of clinical, biomechanical, and radiologic studies. Am J Orthop (Belle Mead NJ) 1999;28:336-40.

15. Lund T, Oxland TR. Adjacent level disk disease: is it really a fusion disease? Orthop Clin North Am 2011;42:529-41.

16. Pfirrmann CW, Metzdorf A, Zanetti M, Hodler J, Boos N. Magnetic resonance classification of lumbar intervertebral disc degeneration. Spine (Phila $\mathrm{Pa}$ 1976) 2001;26:1873-8.

17. Jim JJ, Noponen-Hietala N, Cheung KM, et al. The TRP2 allele of COL9A2 is an age-dependent risk factor for the development and severity of intervertebral disc degeneration. Spine (Phila $\mathrm{Pa} 1976$ ) 2005;30:2735-42.

18. Siemionow K, An H, Masuda K, Andersson G, CsSzabo G. The effects of age, sex, ethnicity, and spinal level on the rate of intervertebral disc degeneration: a review of 1712 intervertebral discs. Spine (Phila Pa 1976) 2011;36:1333-9.

19. Waris E, Eskelin M, Hermunen H, Kiviluoto O, Paajanen H. Disc degeneration in low back pain: a 17year follow-up study using magnetic resonance imaging. Spine (Phila Pa 1976) 2007;32:681-4.

20. Battie MC, Videman T, Parent E. Lumbar disc degeneration: epidemiology and genetic influences. Spine (Phila Pa 1976) 2004;29:2679-90.

21. Luoma K, Vehmas T, Raininko R, Luukkonen R, Riihimaki H. Lumbosacral transitional vertebra: relation to disc degeneration and low back pain. Spine (Phila Pa 1976) 2004;29:200-5.

22. Aihara T, Takahashi K, Ono Y, Moriya H. Does the morphology of the iliolumbar ligament affect lumbosacral disc degeneration? Spine (Phila Pa 1976) 2002;27:1499-503.

23. Aihara T, Takahashi K, Ogasawara A, Itadera E, Ono 
Y, Moriya H. Intervertebral disc degeneration associated with lumbosacral transitional vertebrae: a clinical and anatomical study. J Bone Joint Surg Br 2005;87:687-91.

24. Quinnell RC, Stockdale HR. The use of in vivo lumbar discography to assess the clinical significance of the position of the intercrestal line. Spine (Phila Pa 1976) 1983;8:305-7.

25. Battie MC, Videman T, Gibbons LE, Fisher LD, Manninen H, Gill K. 1995 Volvo Award in clinical sciences: determinants of lumbar disc degeneration: a study relating lifetime exposures and magnetic resonance imaging findings in identical twins. Spine (Phila Pa 1976) 1995;20:2601-12.

26. Videman T, Battie MC, Gill K, Manninen H, Gibbons LE, Fisher LD. Magnetic resonance imaging findings and their relationships in the thoracic and lumbar spine: insights into the etiopathogenesis of spinal degeneration. Spine (Phila Pa 1976) 1995;20:928-35.
27. Natarajan RN, Andersson GB. Lumbar disc degeneration is an equally important risk factor as lumbar fusion for causing adjacent segment disc disease. J Orthop Res 2017;35:123-30.

28. Kong MH, Morishita Y, He W, et al. Lumbar segmental mobility according to the grade of the disc, the facet joint, the muscle, and the ligament pathology by using kinetic magnetic resonance imaging. Spine (Phila Pa 1976) 2009;34:2537-44.

29. Cheung KM, Samartzis D, Karppinen J, et al. Intervertebral disc degeneration: new insights based on "skipped" level disc pathology. Arthritis Rheum 2010;62:2392-400.

30. Hsu K, Zucherman J, Shea W, et al. High lumbar disc degeneration: incidence and etiology. Spine (Phila Pa 1976) 1990;15:679-82. 